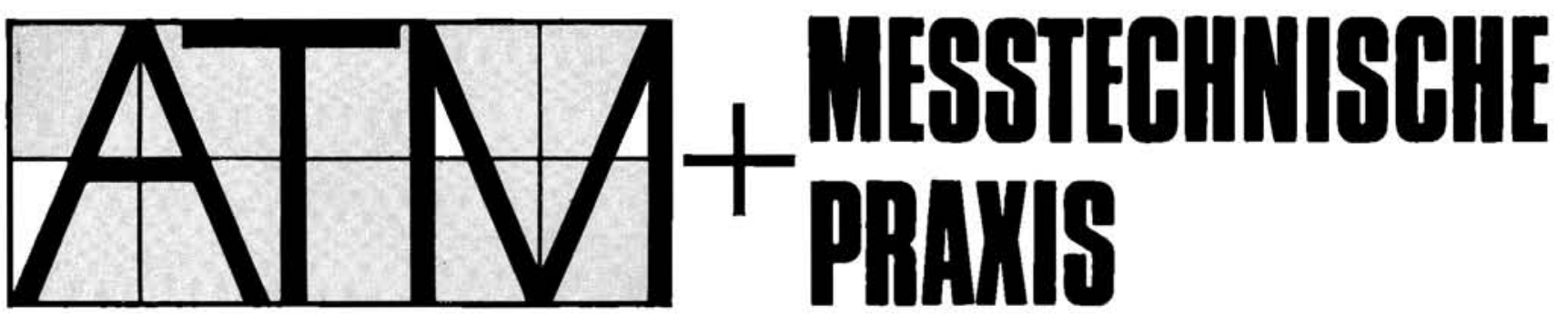

Begründet von : Professor Dr.-ing. Georg Keinath

Herausgeber: Dr.-Ing. Heinrich Toeller

Schriftleitung für ATM Archiv für technisches Messen:

Schriftleitung für die Meßtechnische Praxis:

Verlag:
Professor Dr.-Ing. Karl Walter Bonfig, Lehrstuhl für Meß- und Regelungstechnik, Ruhr-Universität. 463 Bochum-Querenburg, Universitätsstraße 150

Dipl.-Ing. H. R. Eggers, 5628 Heiligenhaus, Rieskuhlstraße 6

\title{
Inhaltsverzeichnis - Januar 1975 - Lieferung 468
}

\section{Meßtechnische Praxis}

Meßtechnik und Prozeßautomatisierung gesehen vom ZVEI-FV 15. Interview mit dem Vorsitzenden Prof. Dr. Herbert Matuschka . . . . . . . .

Measurement engineering and process automation seen from ZVEI-FV 15. Interview with chairman Prof. Dr. Herbert Matuschka

K. Bauer .

Die Errichtung explosionsgeschützter elektrischer Instrumentierungen

Installation of explosion protected electrical instrumentations

Gerätetechnik . . . . . . . . . . R 7

Persönliches - VDI/VDE . . . . . . . R 14

Vorschau auf den Inhalt des Februarheftes, Lieferung 469 H. Ruthmann, Instrumentierung einer Hochofenanlage mit 10000 Tagestonnen

R. Oldenbourg Verlag GmbH, 8 München 80, Rosenheimer Str. 145 Verantwortlich für den Anzeigenteil: K. Haslbeck, München; z. Z gil Anzeigenpreisliste Nr. 24. Erscheinungsweise: 12mal im Jahr. JahresAnzeigenpreisliste $\mathrm{Nr}$. 24. Erscheinungsweise: $12 \mathrm{mal}$ im Jahr. Jahres-
bezugspreis (In- und Ausland): DM 88,- einschlieBlich Mehrwertsteuer zuzüglich DM 6,- Versandspesen (ohne Mehrwertsteuer). - Einzelheft: DM 9,- einschlieBlich Mehrwertsteuer zuzüglich DM -,50 Versandspesen (ohne Mehrwertsteuer). Abbestellungen nur jeweils am Ende eines Kalenderjahres möglich; sie müssen 6 Wochen vorher beim Verlag eintreffen. - Die in dieser Zeitschrift veröffentlichten Beitrăge sind urheberrechtlich geschützt. Ubersetzung, Nachdruck - auch von Abbildungen Verviellältigung ouf photomechanischem oder ahnlichem Wege oder -, Mervielfiligung Magnettonverfahren, Vortrag, Funk-und Fernsehsendung sowie Speicherung in Datenverarbeitungsanlagen - auch auszugsweise - bleiben vorbehalten. Werden von einzelnen Beiträgen oder Teilen von ihnen einzelne Vervielfăltigungsstücke im Rahmen des \$ 54 UrhG hergestellt und dienen diese gewerblichen $Z$ wecken, ist dafür eine Vergütung gem. den gleichlautenden Gesamtvertrãgen zwischen der Verwertungsgesellschaft Wissenschaft $\mathrm{GmbH}$ (ehemals Inkassostelle für urheberrechtliche Vervielfaltigungsgebühren GmbH), 6 Frankfurt/Main, Großer Hirschgraben 17-21, und dem Bundesverband der Deutschen Industrie e.V. dem Gesamtund verband der Banken e.V. dem Dentschen Sparkassen- und Giroverband un Verband der Privaten Bausparkassen e.V. an die VG Wissenschaft zu entrichten. Die Vervielfältigungen sind mit einem Vermerk über die Quelle und den Vervielfältiger zu versehen. Erfolgt die Entrichtung der Gebühren durch Wertmarken der VG Wissenschaft, so ist für jedes vervielfältigte Blatt eine Marke im Wert von DM 0,40 zu verwenden. Druck: $R$. Oldenbourg, Graphische Betriebe GmbH, 8 München 80, Rosenheimer Str. 145. Zeitungsvertriebsamt für die Deutsche Demokratische Republik: X 1004 Berlin, Straße der Pariser Kommune 3-4.

Textbeiträge (Erstveröfentlichungen), die in das Gebiet des Lie-

Textbeitres lallen, sind an die Schrifteitung zu senden. Fu langt eingesandte Manuskripte wird keine Haftung übernommen.

Alleingesellschafter ist $R$. Oldenbourg, München. Die Firma R. Olden bourg ist an der R. Oldenbourg Verlag GmbH außerdem als stille Gesellschafterin beteiligt.

\section{ATM - Archiv für technisches Messen}

V 332-32 H. Galster. . . . . . . . . . . . .

Die Elektronenaktivität als Maß für das Redox-Potential in wäßrigen Lösungen

Electron activity as a measure of Redoxpotential in diluted solutions

V 35192-12 P. Stefán . . . . . . . . . . . . . Eine neue, allgemeine Methode zur Messung des spezifischen Erdbodenwiderstandes

A new general method for measurement of the specific soil resistance

V 354-9 $\quad$ B. Hinrichs . . . . . . . . . . . .

Messung kleiner Verlustfaktoränderungen bei $50 \mathrm{~Hz}$ bei gleichzeitiger Teilentladungsmessung an festen Isolierstoffen bis zum Durchschlag

Measurement of small changes in the loss factor at $50 \mathrm{cps}$ with synchronous measurement of the partial discharge at solid insulation materials until break down J 021-24 H. Walcher . . . . . . . . . . Steigerung der Auswertesicherheit von Liniendiagrammen durch gleitende Integration

Increase in the safety of analysis of line diagrams by sliding integration

J $030-6 \quad$ S. Skoczowski ............ Die theoretischen und praktischen Gesichtspunkte zur Aufzeichnungslesbarkeit von analogen Registriergeräten

Theoretical and practical viewpoints for the legibility of plots of analog registration devices

Vorschau auf den Inhalt des Februarheftes, Lieferung 469

V 365-9 A. Metal und A. Zuchowski Dynamische Meßtechnik-Begriffe und Definitionen V 952-2 H. W. Schöpgens und A. A. Raible Ein schnelles, elektronisch ortskompensierendes Pendelwägesystem hoher Präzision für magnetische Messungen
J 086-2
H. Unbehauen

Einsatz eines Prozeßrechners zur ,on-line“-Messung des dynamischen Verhaltens von Systemen mit Hilfe der Kreuzkorrelationsmethode

J 131-15 U. Trilling

Mathematisches Modell für das Zeitverhalten von Neigungswaagen, Teil I 